\title{
Myocardial fibrosis by CMR LGE in a large cohrt of pediatric thalassemia major patients
}

\author{
Antonella Meloni ${ }^{1 *}$, Maddalena Casale ${ }^{2}$, Aldo Filosa², Blandina Pagano ${ }^{3}$, Vincenzo Positano ${ }^{1}$, Antonino Vallone ${ }^{4}$, \\ Gianluca Valeri ${ }^{5}$, Daniele De Marchi ${ }^{1}$, Massimo Lombardi ${ }^{1}$, Alessia Pepe ${ }^{1}$
}

From 17th Annual SCMR Scientific Sessions

New Orleans, LA, USA. 16-19 January 2014

\section{Background}

Cardiovascular Magnetic Resonance (CMR) by late gadolinium enhancement (LGE) allows to detect myocardial fibrosis. Myocardial fibrosis was shown to be a relative common finding in large cohort of Italian thalassemia major (TM) patients mainly related to $\mathrm{HCV}$ infection, but specific studies involving only pediatric patients are not available. Our aim was to investigate the prevalence and clinical-instrumental correlates of myocardial fibrosis in pediatric TM patients.

\section{Methods}

We studied retrospectively 76 pediatric patients with TM (44 boys, $4.2-17.9$ years old, mean age $13.6 \pm 3.4$ years) enrolled in the MIOT (Myocardial Iron Overload in
Thalassemia) Network. All patients were well transfused and chelated since the early childhood.LGE images were acquired to detect myocardial fibrosis. Myocardial iron overload (MIO) was measured by $\mathrm{T}^{*}$ multislice multiecho technique. Biventricular function parameters were quantitatively evaluated by cine images.

\section{Results}

Myocardial fibrosis was detected in 12 (15.8\%) patients. In all patients the location of the fibrosis was epi-mesocardial, with no ischemic pattern. The youngest patient showing myocardial fibrosis had 13 years of age. Table 1 shows the comparison between patients with and without myocardial fibrosis. A significant higher MIO was detected in patients with myocardial fibrosis. The left atrial area, all the left

Table 1 Clinical and instrumental correlates in the fibrosis and no-fibrosis group.

\begin{tabular}{|c|c|c|c|}
\hline & $\begin{array}{l}\text { Fibrosis } \\
\text { group } \\
(\mathrm{N}=12)\end{array}$ & $\begin{array}{c}\text { No-fibrosis } \\
\text { group } \\
(\mathrm{N}=64)\end{array}$ & P-value \\
\hline $\operatorname{Sex}(M / F)$ & $10 / 2$ & $34 / 30$ & 0.062 \\
\hline Age (years) & $15.4 \pm 1.8$ & $13.3 \pm 3.5$ & 0.073 \\
\hline Transfusions starting age (years) & $1.2 \pm 0.9$ & $1.3 \pm 0.8$ & 0.691 \\
\hline Chelation starting age (years) & $3.1 \pm 1.8$ & $3.1 \pm 2.3$ & 0.705 \\
\hline HCV antibodies, $N(\%)$ & 0 & $3(4.8 \%)$ & 0.437 \\
\hline Hb pre-transfusion ( $g / d l)$ & $9.7 \pm 0.3$ & $9.5 \pm 0.7$ & 0.757 \\
\hline Ferritin levels (ng/l) & $3012 \pm 2167$ & $2225 \pm 1396$ & 0.226 \\
\hline$A L T(u / l)$ & $41.6 \pm 12.5$ & $38.6 \pm 32.6$ & 0.268 \\
\hline AST (u/l) & $46.6 \pm 41.2$ & $33.4 \pm 25.9$ & 0.207 \\
\hline Global Heart T2* (ms) & $20.9 \pm 13.9$ & $30.6 \pm 9.7$ & 0.022 \\
\hline
\end{tabular}

${ }^{1}$ CMR Unit, Fondazione G.Monasterio CNR-Regione Toscana and Institute of

Clinical Physiology, Pisa, Italy

Full list of author information is available at the end of the article 
Table 1 Clinical and instrumental correlates in the fibrosis and no-fibrosis group. (Continued)

\begin{tabular}{|c|c|c|c|}
\hline MRI CIC (mg/g dry weight) & $2.0 \pm 1.7$ & $0.8 \pm 0.6$ & 0.022 \\
\hline Patients with global heart T2* $<20 \mathrm{~ms}, N(\%)$ & $7(58.3)$ & $12(18.8)$ & 0.008 \\
\hline N. of seg. with abnormal T2* & $9.0 \pm 7.0$ & $3.8 \pm 5.2$ & 0.030 \\
\hline Left atrial area $(\mathrm{cm} 2)$ & $18.3 \pm 3.1$ & $15.9 \pm 3.9$ & 0.050 \\
\hline Right atrial area $(\mathrm{cm} 2)$ & $16.9 \pm 4.3$ & $14.9 \pm 3.5$ & 0.169 \\
\hline Left ventricular end-diastolic volume index (m//m2) & $102.9 \pm 23.5$ & $87.0 \pm 16.3$ & 0.005 \\
\hline Left ventricular end-systolic volume index $(\mathrm{m} / \mathrm{m} 2)$ & $42.0 \pm 12.1$ & $35.1 \pm 8.9$ & 0.022 \\
\hline Left ventricular stroke volume index $(\mathrm{m} / \mathrm{m} 2)$ & $60.7 \pm 12.4$ & $51.8 \pm 10.7$ & 0.012 \\
\hline Left ventricular mass index $(\mathrm{g} / \mathrm{m} 2)$ & $65.3 \pm 11.4$ & $53.8 \pm 11.4$ & 0.003 \\
\hline Left ventricular ejection fraction (\%) & $59.2 \pm 4.4$ & $59.7 \pm 5.9$ & 0.368 \\
\hline Right ventricular end-diastolic volume index $(\mathrm{m} / / \mathrm{m} 2)$ & $96.9 \pm 25.6$ & $81.6 \pm 17.1$ & 0.089 \\
\hline Right ventricular end-systolic volume index ( $\mathrm{ml} / \mathrm{m} 2)$ & $36.9 \pm 13.7$ & $32.3 \pm 8.3$ & 0.458 \\
\hline Right ventricular stroke volume index $(\mathrm{ml} / \mathrm{m} 2)$ & $61.5 \pm 11.6$ & $48.9 \pm 14.1$ & 0.005 \\
\hline Right ventricular ejection fraction (\%) & $62.6 \pm 4.4$ & $60.2 \pm 7.1$ & 0.175 \\
\hline
\end{tabular}

ventricular (LV) indexed volumes, the LV mass index and the bi-ventricular stroke volume indexes were significantly higher in the fibrosis group than in the no-fibrosis group.

\section{Conclusions}

In pediatric TM patients myocardial fibrosis is not a rare finding to keep in mind in the cardiological management. When appropriate treatment has been administered since early childhood, CMR LGE can be postponed until 13 years of age. By the natural history of this large cohort of pediatric patients where $\mathrm{HCV}$ infection has been appropriately prevented, myocardial fibrosis seem to be associated with MIO and high cardiac output.

\section{Funding}

The MIOT project receives "no-profit support" from industrial sponsorships (Chiesi Farmaceutici S.p.A. and ApoPharma Inc.). This study was also supported by: "Ministero della Salute, fondi ex art. 12 D.Lgs. 502/92 e s.m.i., ricerca sanitaria finalizzata anno 2006" and "Fondazione $\mathrm{L}$. Giambrone".

\footnotetext{
Authors' details

${ }^{1}$ CMR Unit, Fondazione G.Monasterio CNR-Regione Toscana and Institute of Clinical Physiology, Pisa, Italy. ${ }^{2}$ Centro per le Microcitemie, AORN Cardarelli, Napoli, Italy. ${ }^{3}$ Centro Microcitemico, U.O. di Pediatria e Neonatologia. Presidio Ospedaliero Locri - A.S.L. n. 9, Locri, Italy. ${ }^{4}$ Istituto di Radiologia, Az. Osp. "Garibaldi" Presidio Ospedaliero Nesima, Catania, Italy. ${ }^{5}$ Dipartimento di Radiologia, Azienda Ospedaliero-Universitaria Ospedali Riuniti "Umberto ILancisi-Salesi", Ancona, Italy.
}

Published: 16 January 2014

doi:10.1186/1532-429X-16-S1-P395

Cite this article as: Meloni et al:: Myocardial fibrosis by CMR LGE in a large cohrt of pediatric thalassemia major patients. Journal of

Cardiovascular Magnetic Resonance 2014 16(Suppl 1):P395.
Submit your next manuscript to BioMed Central and take full advantage of:

- Convenient online submission

- Thorough peer review

- No space constraints or color figure charges

- Immediate publication on acceptance

- Inclusion in PubMed, CAS, Scopus and Google Scholar

- Research which is freely available for redistribution

Submit your manuscript at www.biomedcentral.com/submit 